\title{
Gender difference in the association between food away-from-home consumption and body weight outcomes among Chinese adults
}

\author{
Wen-Wen Du, Bing Zhang*, Hui-Jun Wang, Zhi-Hong Wang, Chang Su, Ji-Guo Zhang, \\ Ji Zhang, Xiao-Fang Jia and Hong-Ru Jiang \\ National Institute for Nutrition and Health, Chinese Center for Disease Control and Prevention, 29 Nanwei Road, \\ Xicheng District, Beijing 100050, People's Republic of China
}

Submitted 9 November 2015: Final revision received 16 April 2016: Accepted 25 April 2016: First published online 26 May 2016

\begin{abstract}
Objective: The present study aimed to explore the associations between food away-from-home (FAFH) consumption and body weight outcomes among Chinese adults.

Design: FAFH was defined as food prepared at restaurants and the percentage of energy from FAFH was calculated. Measured BMI and waist circumference (WC) were used as body weight outcomes. Quantile regression models for BMI and WC were performed separately by gender.

Setting: Information on demographic, socio-economic, diet and health parameters at individual, household and community levels was collected in twelve provinces of China.

Subjects: A cross-sectional sample of 7738 non-pregnant individuals aged 18-60 years from the China Health and Nutrition Survey 2011 was analysed.

Results: For males, quantile regression models showed that percentage of energy from FAFH was associated with an increase in BMI of 0.01, 0.01, 0.01, 0.02, 0.02 and $0.03 \mathrm{~kg} / \mathrm{m}^{2}$ at the $5 \mathrm{th}, 25 \mathrm{th}, 50 \mathrm{th}, 75 \mathrm{th}, 90 \mathrm{th}$ and $95 \mathrm{th}$ quantile, and an increase in WC of $0.04,0.06,0.06,0.04,0.06,0.05$ and $0.07 \mathrm{~cm}$ at the 5 th, 10th, 25th, 50th, 75th, 90th and 95th quantile. For females, percentage of energy from FAFH was associated with $0 \cdot 01,0 \cdot 01,0.01$ and $0.02 \mathrm{~kg} / \mathrm{m}^{2}$ increase in BMI at the 10 th, 25 th, 90th and 95th quantile, and with $0.05,0.04,0.03$ and $0.03 \mathrm{~cm}$ increase in WC at the 5th, 10th, 25th and 75th quantile.

Conclusions: Our findings suggest that FAFH consumption is relatively more important for BMI and WC among males rather than females in China. Public health initiatives are needed to encourage Chinese adults to make healthy food choices when eating out.
\end{abstract}

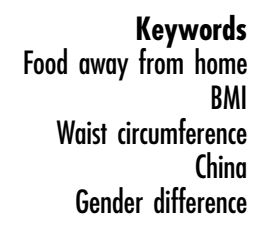

China has experienced a significant increase in the prevalence of obesity during recent decades ${ }^{(1,2)}$, with increasing BMI and waist circumference (WC) over time, particularly for those at the highest BMI and WC levels ${ }^{(3)}$. The increasing obesity epidemic has also shown gender disparities in terms of sociodemographic characteristics. For example, obesity prevalence was higher in lower educated women, but in men with higher education ${ }^{(4)}$.

One of the major factors underlying the obesity epidemic is considered to be the dramatic transition in dietary intake and eating behaviours in China. Previous work has identified higher fat and sodium intakes, accompanied by increasing consumption of edible oils and animal-source foods, and prevalent snacking and eating out ${ }^{(5)}$. Typically, food away from home (FAFH) is defined as food eaten or prepared outside the home, including restaurant food, which comprises the majority of FAFH, as well as foods obtained from canteens and fixed or mobile stalls. In China, consumption of FAFH has increased substantially. The share of urban food expenditure on FAFH rose from $14.7 \%$ in 2000 to $20.6 \%$ in $2008^{(6)}$. A large body of literature has shown that FAFH consumption is associated with high intakes of energy and saturated fat, and low micronutrient intakes ${ }^{(7-10)}$.

Meanwhile, other studies in developed countries have reported a relationship between increased FAFH consumption and higher BMI or risk of obesity, which varied by away-from-home location ${ }^{(11-13)}$. However, most previous studies focused on the association of FAFH with weight-related indicators evaluated at the mean level, 
which do not take into account different conditions across the entire distribution of weight-related indicators. For example, the extent of the association between FAFH and BMI or WC for individuals at the bottom quantiles may differ from that for individuals at the upper quantiles ${ }^{(12)}$. It is especially unclear how FAFH consumption affects body weight outcomes across the distribution among Chinese adults when considering the gender difference.

The present study explored gender disparities in the associations between FAFH consumption and two body weight outcomes among Chinese adults aged 18-60 years, using the latest cross-sectional data from a large-scale cohort study, in order to depict the role of FAFH in the growing prevalence of obesity in a developing country.

\section{Methods}

The data used in the present study were drawn from the 2011 China Health and Nutrition Survey (CHNS), which is a longitudinal, household-based study that began in $1989^{(14)}$. The CHNS included eight or nine diverse provinces differing in terms of economic development and geography in the first eight waves (1989, 1991, 1993, 1997, 2000, 2004, 2006, 2009) and an additional three megacities (Beijing, Shanghai, Chongqing) in the latest 2011 wave. A multistage cluster random sampling method was used to derive the original sample. Counties in each province were stratified by income (low, middle and high) and a weighted sampling scheme was used to randomly select four counties. In addition, the capital and a lowerincome city of a province were selected when feasible, except that other large cities rather than capitals had to be selected in two provinces. Four villages and townships within the counties and urban/suburban neighbourhoods within the cities were selected randomly. Twenty households were selected randomly in each candidate community. Dimensions of data collected in the CHNS included demographics, socio-economics, diet, physical activity and health from individual, household and community levels. Details of the project are described elsewhere $^{(15)}$.

Our analysis included non-pregnant individuals aged 18-60 years in the 2011 survey with complete dietary records and weight, height and WC measurements. Participants who reported having been diagnosed with non-communicable chronic diseases were excluded because they may have strict limitations on eating behaviours and weight control ( $n$ 837). A final sample of 7738 adults (3604 males and 4134 females) was available.

The CHNS provided detailed individual food intake information through in-person interviewer-administered $24 \mathrm{~h}$ recalls conducted by trained staff over three consecutive days, including two working days and one weekend day. For each ingredient of foods consumed in the period, details were collected on the amount consumed, the time of the meal, dining place, and preparation method and location. The energy and nutritional contents of reported food items were coded according to the China Food Composition Table. Household condiment consumption was determined by examining changes from the beginning to the end of each day during the aforementioned $3 \mathrm{~d}$. All condiments (including edible oils) remaining after the last meal before initiation of the survey were weighed and recorded. Condiments brought into the household unit and wasted were estimated in the next $3 \mathrm{~d}$. At the end of the dietary survey, all remaining condiments were again weighed and recorded. The number of household members and visitors was recorded at each meal across $3 \mathrm{~d}$. Energy intake from condiments was divided among members and visitors by proportion of total food intake in households. Total energy intake was summed as energy from individual reported foods and energy from weighed household condiments, mainly cooking oil. All food items in a mealtime that were prepared away from home regardless of dining place were defined as $\mathrm{FAFH}^{(8,16,17)}$. In the present study, FAFH specifically referred to food prepared at restaurants, including fast-food restaurants, full-service restaurants and outdoor fixed food stalls. Food prepared at friends' or relatives' home was counted as food at-home consumption. Then we calculated energy from total FAFH and derived the percentage of energy intake from FAFH.

Field-measured height, weight and WC were used for analysis. BMI was calculated as weight divided by squared height $\left(\mathrm{kg} / \mathrm{m}^{2}\right)$. Anthropometric measurements were conducted by trained local staff according to protocols of the $\mathrm{WHO}^{(18,19)}$. We used a stadiometer (SECA 206) and electronic weight scales (SECA 882) for height and weight measurement, respectively. A measuring tape was used for WC measurement.

Demographic, socio-economic, diet and lifestyle variables were controlled in the models as covariates, including age (years), highest level of education attained (primary school and below $=0$, junior school $=1$, senior school and above $=2$ ), per capita annual household income level (low tertile $=0$, middle tertile $=1$, high tertile $=2$ ), urbanization level of resident community (low tertile $=0$, middle tertile $=1$, high tertile $=2$ ), daily energy intake (kJ/day), current smoking (yes/no), drinking alcohol over the last year (yes/no) and work-related moderateto-vigorous physical activity (yes/no). The information was collected by questionnaire survey on individual, household and community levels. More definitions of above variables can be found on the website (http://www.cpc.unc.edu/ projects/china/data/questionnaires).

Descriptive analyses were conducted to investigate the distribution of main variables. According to the multilevel characteristics of eating behaviours and body weight outcomes, we performed series of quantile regression models for continuous BMI and WC for males and females, respectively, to investigate the varied impacts of 
percentage of energy from FAFH on different quantiles of body weight outcomes. In model 1, we only included percentage of energy from $\mathrm{FAFH}$ in the quantile regression model to investigate the association with BMI/WC. Model 2 controlled for additional individual variables (age, total energy intake, education level, smoking, drinking and moderate-to-vigorous physical activity). Model 3 additionally included the household variable (per capita annual household income level); model 4 controlled for the community variable (urbanization level) besides aforementioned covariates. All statistical analyses were performed using the statistical software package SAS version 9.2. The quantile regression was estimated using the PROC QUANTREG procedure.

\section{Results}

Summary statistics of variables used in the present study are presented in Table 1. Mean and median values for continuous variables and percentages for category variables are shown. The average BMI and WC was $23.6 \mathrm{~kg} / \mathrm{m}^{2}$ and $82.6 \mathrm{~cm}$ in the participants, mean age 42.9 years, respectively. The energy attributed to FAFH was $1357 \mathrm{~kJ} / \mathrm{d}$ $(324.4 \mathrm{kcal} / \mathrm{d})$ in total, $1624 \mathrm{~kJ} / \mathrm{d}(388.1 \mathrm{kcal} / \mathrm{d})$ in males and $1124 \mathrm{~kJ} / \mathrm{d}(268.7 \mathrm{kcal} / \mathrm{d})$ in females, which contributed $15.4 \%$ to total dietary energy intake in total, $16.9 \%$ in males and $14.2 \%$ in females.

The estimates for FAFH consumption in the distribution of BMI are shown in Fig. 1. For males (Fig. 1(a)), the quantile regression coefficients for the percentage of energy from FAFH across all BMI quantiles were statistically significant. For females (Fig. 1(b)), significant coefficients for the percentage of energy from $\mathrm{FAFH}$ were observed only at the 10th, 25th, 90th and 95th BMI quantiles. Moreover, the increases in BMI were higher at the upper $v$. the lower end of the BMI distribution, indicating that heavier males and females got larger increases in BMI with an additional percentage of energy from FAFH, compared with individuals with lower body mass. Increases in BMI attributed to an additional percentage of energy from FAFH across the quantiles were $0.01-0.03 \mathrm{~kg} / \mathrm{m}^{2}$ in males and $0 \cdot 01-0.02 \mathrm{~kg} / \mathrm{m}^{2}$ in females.

The quantile regression estimates for FAFH consumption in the distribution of WC are displayed in Fig. 2. For males (Fig. 2(a)), similar to the findings for BMI, we observed a significant increase in WC with additional FAFH consumption across all WC quantiles. For females (Fig. 2(b)), the quantile regression coefficients for FAFH consumption at the 5th, 10th, 25th and 75th WC quantiles were statistically significant. However, significant associations were not observed at the other quantiles. The increases in WC were higher at the lower than the upper end of the WC distribution, indicating that females with lower WC experienced larger increases in WC with an additional percentage of energy from FAFH, compared with individuals with higher WC.

Adjusted associations between FAFH consumption and body weight outcomes in males and females were estimated, respectively, using quantile regression, as shown in Table 2. In general, the effects of FAFH on body

Table 1 Demographic characteristics of the sample of non-pregnant individuals aged 18-60 years, China Health and Nutrition Survey 2011

\begin{tabular}{|c|c|c|c|c|c|c|c|c|c|}
\hline \multirow[b]{2}{*}{ Variable } & \multicolumn{3}{|c|}{ Males (n 3604) } & \multicolumn{3}{|c|}{ Females ( $n$ 4134) } & \multicolumn{3}{|c|}{ Total ( $n 7738)$} \\
\hline & Mean & SD & Median & Mean & SD & Median & Mean & SD & Median \\
\hline Age (years) & 42.9 & $11 \cdot 1$ & 44.0 & $42 \cdot 8$ & $10 \cdot 9$ & $44 \cdot 0$ & 42.9 & $11 \cdot 0$ & $44 \cdot 0$ \\
\hline BMl $\left(\mathrm{kg} / \mathrm{m}^{2}\right)$ & 23.8 & 3.5 & 23.5 & 23.5 & 3.9 & $23 \cdot 1$ & 23.6 & 3.7 & $23 \cdot 3$ \\
\hline Waist circumference (cm) & $85 \cdot 3$ & 10.4 & $85 \cdot 0$ & $80 \cdot 1$ & 9.9 & 80.0 & 82.6 & 10.5 & $82 \cdot 0$ \\
\hline Total energy intake (kJ/d) & 9285 & 3059 & 8986 & 7784 & 2597 & 7477 & 8483 & 2919 & 8151 \\
\hline Total energy intake $(\mathrm{kcal} / \mathrm{d})$ & $2219 \cdot 2$ & $731 \cdot 0$ & $2147 \cdot 7$ & $1860 \cdot 4$ & $620 \cdot 7$ & $1787 \cdot 0$ & $2027 \cdot 5$ & 697.6 & $1948 \cdot 2$ \\
\hline Energy intake from FAFH (kJ/d) & 1624 & 1997 & 1120 & 1124 & 1443 & 664 & 1357 & 1741 & 898 \\
\hline Energy intake from FAFH (kcal/d) & $388 \cdot 1$ & $477 \cdot 2$ & $267 \cdot 8$ & 268.7 & 344.9 & $158 \cdot 7$ & $324 \cdot 3$ & $416 \cdot 1$ & $214 \cdot 7$ \\
\hline$\%$ of energy from FAFH & $16 \cdot 9$ & $19 \cdot 4$ & $12 \cdot 8$ & $14 \cdot 2$ & $17 \cdot 3$ & $9 \cdot 2$ & $15 \cdot 4$ & $18 \cdot 3$ & $10 \cdot 8$ \\
\hline \multicolumn{10}{|l|}{ Per capita household income (Yuan/year) } \\
\hline Low & $4477 \cdot 4$ & $2743 \cdot 5$ & $4624 \cdot 4$ & $4312 \cdot 1$ & $2738 \cdot 9$ & $4463 \cdot 2$ & 4384.5 & $2741 \cdot 6$ & $4537 \cdot 6$ \\
\hline Middle & $12253 \cdot 1$ & 2972.5 & 12074.0 & $12333 \cdot 7$ & $3030 \cdot 7$ & $12137 \cdot 3$ & $12295 \cdot 4$ & 3002.9 & $12091 \cdot 3$ \\
\hline High & $27360 \cdot 5$ & 9259.5 & 24795.4 & $27103 \cdot 8$ & $9011 \cdot 3$ & $24667 \cdot 7$ & $27228 \cdot 1$ & 9131.5 & 24731.6 \\
\hline \multicolumn{10}{|l|}{ Urbanization index } \\
\hline Low & $51 \cdot 8$ & $11 \cdot 3$ & $50 \cdot 2$ & $51 \cdot 6$ & $11 \cdot 3$ & $49 \cdot 8$ & $51 \cdot 7$ & $11 \cdot 3$ & $50 \cdot 2$ \\
\hline Middle & $74 \cdot 1$ & $10 \cdot 9$ & $72 \cdot 2$ & 74.6 & $11 \cdot 0$ & $72 \cdot 2$ & $74 \cdot 3$ & 10.9 & $72 \cdot 2$ \\
\hline High & $92 \cdot 3$ & $5 \cdot 4$ & $92 \cdot 6$ & $92 \cdot 4$ & 5.4 & $92 \cdot 7$ & $92 \cdot 4$ & $5 \cdot 4$ & 92.6 \\
\hline & & $\%$ & & & $\%$ & & & $\%$ & \\
\hline \multicolumn{10}{|l|}{ Education level } \\
\hline Primary school and below & & $19 \cdot 8$ & & & $30 \cdot 4$ & & & 25.5 & \\
\hline Junior school & & $38 \cdot 7$ & & & $33 \cdot 3$ & & & $35 \cdot 8$ & \\
\hline Senior school and above & & 41.5 & & & $36 \cdot 3$ & & & $38 \cdot 7$ & \\
\hline Smoking & & $56 \cdot 7$ & & & 1.6 & & & $27 \cdot 3$ & \\
\hline Drinking alcohol & & 63.4 & & & $12 \cdot 6$ & & & $36 \cdot 2$ & \\
\hline Moderate-to-vigorous physical activity & & $54 \cdot 4$ & & & $35 \cdot 2$ & & & $44 \cdot 2$ & \\
\hline
\end{tabular}

FAFH, food away from home. 

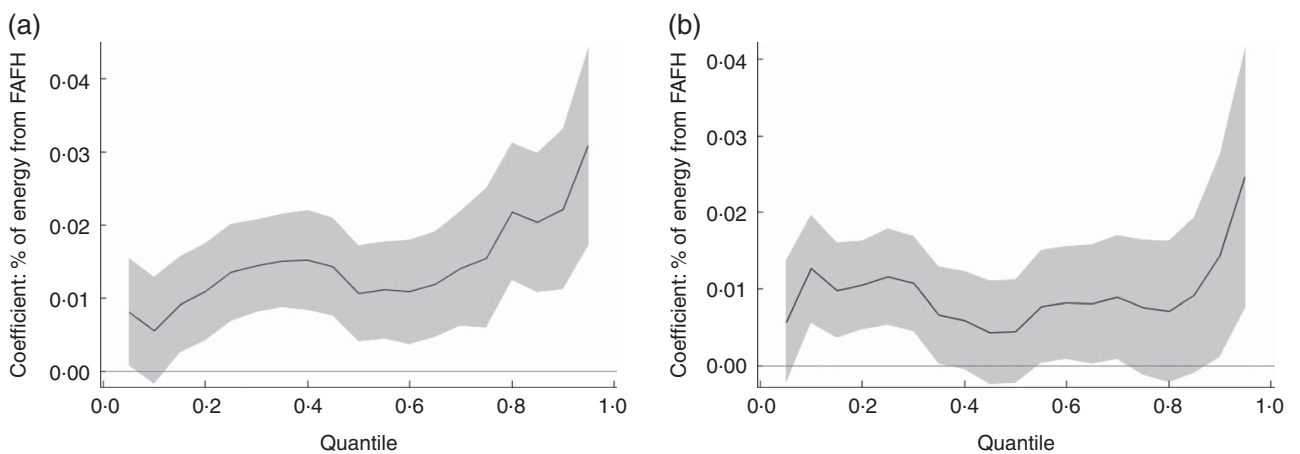

Fig. 1 Association between percentage of energy from food away from home (FAFH) and BMI in quantile regression model, according to gender (a, males; b, females), among non-pregnant individuals ( $n 7738$ ) aged $18-60$ years, China Health and Nutrition Survey 2011. The solid line represents the quantile estimates; the grey area surrounding the solid line represents the $95 \%$ confidence interval for the estimates. All models controlled for age, total energy intake, education level, household income, urbanization level, smoking, drinking and moderate-to-vigorous physical activity
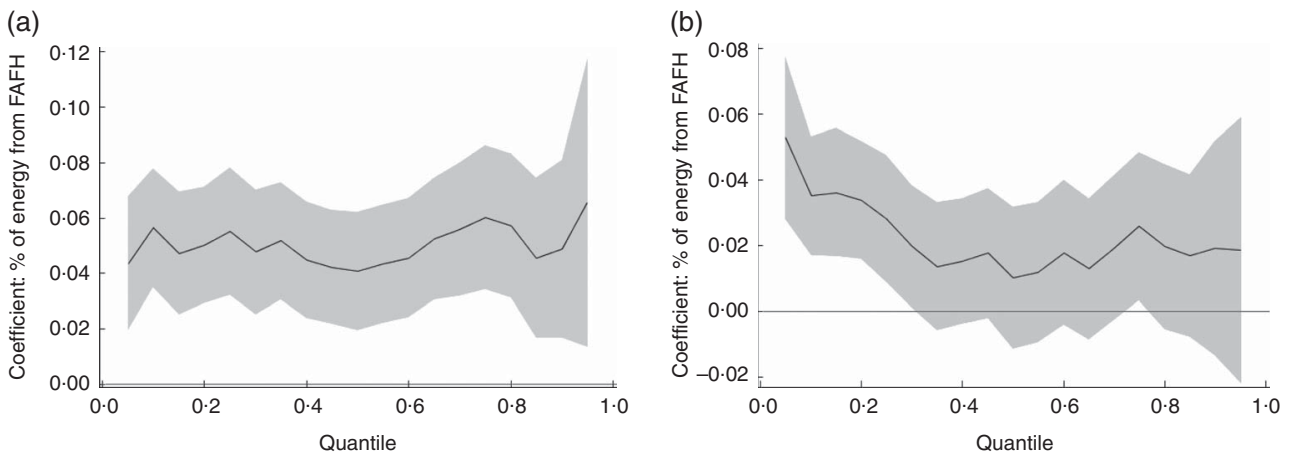

Fig. 2 Association between percentage of energy from food away from home (FAFH) and waist circumference in quantile regression model, according to gender (a, males; b, females), among non-pregnant individuals ( $n$ 7738) aged $18-60$ years, China Health and Nutrition Survey 2011. The solid line represents the quantile estimates; the grey area surrounding the solid line represents the $95 \%$ confidence interval for the estimates. All models controlled for age, total energy intake, education level, household income, urbanization level, smoking, drinking and moderate-to-vigorous physical activity

weight outcomes were weaker for females than males. The extent of the association was larger for WC than for BMI in both genders across the entire distribution. The regression estimates generally increased with the outcomes' quantiles, except for WC in females, which demonstrated a decreasing trend by quantiles. In the context of our findings, an additional $10 \%$ of energy from FAFH will result in an increase of BMI of 0.08 to $0.31 \mathrm{~kg} / \mathrm{m}^{2}$ and an increase in WC of 0.41 to $0.66 \mathrm{~cm}$ across the entire quantiles for males $(P<0.05)$; while in females the corresponding values are 0.12 to $0.25 \mathrm{~kg} / \mathrm{m}^{2}$ increment in BMI and 0.26 to $0.53 \mathrm{~cm}$ increment in WC at the lower and middle-upper quantiles $(P<0.05)$.

\section{Discussion}

The current study evidenced the different associations by gender between FAFH consumption and BMI or WC in adults using data from China Health and Nutrition Survey 2011. In addition, the heterogeneity of the association of
FAFH consumption with the body weight outcomes across the entire conditional distribution of BMI and WC was also observed.

Our results concurred with previous studies that showed higher FAFH consumption may play an important role in the current obesity epidemic for adults. Away-fromhome eating has been shown to be characterized by high energy and fat intakes, especially saturated fat, along with low micronutrient intakes ${ }^{(7-9,20,21)}$, which could explain why more FAFH consumption increases the body weight indicators. A national survey among Korean adults reported that heavy FAFH consumption (obtaining $\geq 5858 \mathrm{~kJ}$ $(\geq 1400 \mathrm{kcal})$ from FAFH per day), specifically at fullservice restaurants, was significantly related to higher BMI and WC, in which larger associations were found at higher quantiles $^{(12)}$. Other studies also have shown that frequent away-from-home meals were related to higher body weight outcomes, such as BMI, WC and weight, or higher risk of becoming overweight/obese ${ }^{(11,22-24)}$. Building on the previous literature in developed countries, the present study explored more evidence on gender disparities in the 
association between FAFH consumption and body weight outcomes using data from China, which could help understanding the effects of nutrition transition on human health in developing countries. As a phenomenon of socio-economic development, eating at restaurants is becoming more popular than in past decades and is beginning to play an important role in the modern diet ${ }^{(20,22,25-28)}$. Restaurant foods provided $15 \cdot 4 \%$ of total energy in the present study, which showed positive associations with higher BMI and WC for Chinese adults.

We found that the associations between FAFH consumption and body weight outcomes were different by gender. Overall, the quantile regression results demonstrated that more FAFH was significantly associated with higher body weight outcomes among males, while the association was not always significant among females throughout the distribution of both outcomes. Our findings further indicated that the magnitude of the association appeared to be larger for males than for females.

Gender disparities in BMI and obesity were reported in previous studies, which illustrated that socio-economic factors may induce more effects on men than women ${ }^{(29,30)}$. Other studies demonstrated that multifaceted causes may contribute to the gender differences in FAFH consumption and body weight outcomes. For example, changes in food prices and income account for half of weight gain for males, but only a small fraction for females ${ }^{(31-33)}$. Meanwhile, gender norms set different roles in the household and status in society. The literature reports that male, unmarried, employed, higher-educated and high-income individuals consume more meals away from home ${ }^{(34)}$. On the other hand, women take more responsibilities in caring for the family in China, which makes them eat out less often than men. Furthermore, females usually prefer to control weight and choose relatively healthy foods when eating out, which may explain the limited association between FAFH consumption and body weight outcomes for females.

Previous studies showed that individuals at high percentiles of the BMI distribution have a greater increase in BMI induced by FAFH than those at low percentiles ${ }^{(30,35)}$. Our results also indicated that the associations between FAFH and body weight outcomes showed higher magnitude in the upper tail of the BMI distribution for both genders. Inconsistent with a Korean study, which found that heavy FAFH consumption had overall larger associations with both BMI and WC at higher quantiles ${ }^{(12)}$, however, the present study indicated the similar finding only on BMI among Chinese adults. Furthermore, FAFH played smoothly positive effects on the entire distribution of $\mathrm{WC}$ for males, while it played stronger effects at the lower tail of the WC distribution for females. Relative to FAFH, other demographic and lifestyle variables may largely contribute to the increase in WC in females at the upper tail of the WC distribution. For the two outcomes, FAFH indicated a larger influence on WC than on BMI among both genders. The findings imply that 
FAFH consumption may increase the risk of larger BMI and WC for Chinese adults, which are established as harmful factors for non-communicable chronic diseases.

Our study has several limitations. First, although the sample included twelve provinces in China covering a wide demographic distribution, the cross-sectional data from CHNS used in present study are not nationally representative; therefore, extrapolated conclusion should be made cautiously. It is also important to note that the cross-sectional analysis does not allow assessment of the causal relationship of FAFH consumption with body weight outcomes. Second, the dietary data were drawn mainly from self-reported detailed dietary recall and in-household weighing, which may not include cooking oil intake when eating away from home and thus may underestimate energy from FAFH. Therefore, the extent of the positive association of FAFH consumption with body weight outcomes may be also underestimated in the present study. Lastly, unlike other studies conducted in Western countries, fast foods are not prevalent in China, so we combined all restaurant foods into one category in the study, which may weaken the heterogeneity of associations of FAFH consumption in different types of restaurant with body weight outcomes.

The present study fills a gap in the literature concerning different associations between FAFH consumption and body weight outcomes in the conditional distribution by estimating quantile regression models among Chinese adults, revealing potential implications for public health policy making.

\section{Conclusions}

In summary, our study highlights the different associations of FAFH consumption with body weight outcomes among Chinese adult men and women. Males are more likely affected by FAFH than females, as a result having higher BMI and WC. More FAFH consumption may result in higher BMI and WC among Chinese adults, with heterogeneity across the distribution of body weight outcomes. Our results imply that efforts considering these gender disparities should be made to promote healthy food choices when eating away from home. These initiatives will help the public to respond effectively to challenges from the away-from-home food environment and the consequent health risk.

\section{Acknowledgements}

Acknowledgements: This research used data from the China Health and Nutrition Survey (CHNS). Financial support: The authors thank the National Institute for Nutrition and Health at the Chinese Center for Disease Control and Prevention, the Carolina Population Center (grant number 5 R24 HD050924), the University of North Carolina at Chapel Hill, the US National Institutes of Health (NIH; grant numbers R01-HD30880, DK056350, R24 HD050924 and R01-HD38700) and the Fogarty International Center, NIH, for providing financial support for the CHNS data collection and analysis of files from 1989 to 2011 and future surveys. The research was also supported by a research grant from the NIH (grant number $1 \mathrm{U} 54$ HD070725-01), the Eunice Kennedy Shriver National Institute of Child Health \& Human Development, and the Office of Behavioral and Social Sciences Research. It is part of the collaboration project between the National Institute of Nutrition and Health at the Chinese Center for Disease Control and Prevention and the University at Buffalo, State University of New York. The funders had no role in the design, analysis or writing of this article. Conflict of interest: All the authors declare no conflict of interest. Authorship: B.Z. conceived and supervised the study. W.-W.D., H.-J.W. and C.S. analysed and interpreted data. W.-W.D. drafted the manuscript and Z.-H.W., J.-G.Z., J.Z., X.-F.J. and H.-R.J. revised it. All authors approved the final version of the manuscript. Ethics of buman subject participation: This research has been approved by the Institutional Review Committees of the University of North Carolina at Chapel Hill and the National Institute for Nutrition and Health, Chinese Center for Disease Control and Prevention. All subjects gave written informed consent for their participation in the survey.

\section{References}

1. Du T, Sun X, Yin P et al. (2013) Increasing trends in central obesity among Chinese adults with normal body mass index, 1993-2009. BMC Public Health 13, 327.

2. Xi B, Liang Y, He T et al. (2012) Secular trends in the prevalence of general and abdominal obesity among Chinese adults, 1993-2009. Obes Rev 13, 287-296.

3. Stern D, Smith LP, Zhang B et al. (2014) Changes in waist circumference relative to body mass index in Chinese adults, 1993-2009. Int J Obes (Lond) 38, 1503-1510.

4. Gordon-Larsen P, Wang H \& Popkin BM (2014) Overweight dynamics in Chinese children and adults. Obes Rev 15, Suppl. 1, 37-48.

5. Zhai FY, Du SF, Wang ZH et al. (2014) Dynamics of the Chinese diet and the role of urbanicity, 1991-2011. Obes Rev 15, Suppl. 1, 16-26.

6. Dong X \& Hu B (2010) Regional difference in food consumption away from home of urban residents: a panel data analysis. Agric Agric Sci Proc 1, 271-277.

7. Bezerra IN, Junior EV, Pereira RA et al. (2015) Away-fromhome eating: nutritional status and dietary intake among Brazilian adults. Public Health Nutr 18, 1011-1017.

8. Guthrie JF, Lin BH \& Frazao E (2002) Role of food prepared away from home in the American diet, 1977-78 versus 1994-96: changes and consequences. J Nutr Educ Behav 34, 140-150.

9. Orfanos P, Naska A, Trichopoulou A et al. (2009) Eating out of home: energy, macro- and micronutrient intakes in 10 European countries. The European Prospective 
Investigation into Cancer and Nutrition. Eur J Clin Nutr $\mathbf{6 3}$, Suppl. 4, S239-S262.

10. Lachat C, Nago E, Verstraeten R et al. (2012) Eating out of home and its association with dietary intake: a systematic review of the evidence. Obes Rev 13, 329-346.

11. Duffey KJ, Gordon-Larsen P, Jacobs DR Jr et al. (2007) Differential associations of fast food and restaurant food consumption with 3-y change in body mass index: the Coronary Artery Risk Development in Young Adults Study. Am J Clin Nutr 85, 201-208.

12. Kim TH, Lee EK \& Han E (2014) Food away from home and body mass outcomes: taking heterogeneity into account enhances quality of results. Nutrition $\mathbf{3 0}$, 1015-1021.

13. Larson N, Neumark-Sztainer D, Laska MN et al. (2011) Young adults and eating away from home: associations with dietary intake patterns and weight status differ by choice of restaurant. J Am Diet Assoc 111, 1696-1703.

14. Zhang B, Zhai FY, Du SF et al. (2014) The China Health and Nutrition Survey, 1989-2011. Obes Rev 15, Suppl. 1, 2-7.

15. Popkin BM, Du S, Zhai F et al. (2010) Cohort profile: the China Health and Nutrition Survey - monitoring and understanding socio-economic and health change in China, 1989-2011. Int J Epidemiol 39, 1435-1440.

16. Lin BH, Wendt M \& Guthrie JF (2013) Impact on energy, sodium and dietary fibre intakes of vegetables prepared at home and away from home in the USA. Public Health Nutr 16, 1937-1943.

17. Adair LS \& Popkin BM (2005) Are child eating patterns being transformed globally? Obes Res 13, 1281-1299.

18. Shankar B (2010) Obesity in China: the differential impacts of covariates along the BMI distribution. Obesity (Silver Spring) 18, 1660-1666.

19. Wang H, Du S, Zhai F et al. (2007) Trends in the distribution of body mass index among Chinese adults, aged 20-45 years (1989-2000). Int J Obes (Lond) 31, 272-278.

20. Bezerra IN, de Moura Souza A, Pereira RA et al. (2013) Contribution of foods consumed away from home to energy intake in Brazilian urban areas: the 2008-9 Nationwide Dietary Survey. Br J Nutr 109, 1276-1283.

21. Gorgulho BM, Fisberg RM \& Marchioni DM (2013) Nutritional quality of major meals consumed away from home in Brazil and its association with the overall diet quality. Prev Med 57, 98-101.

22. Kant AK, Whitley MI \& Graubard BI (2014) Away from home meals: associations with biomarkers of chronic disease and dietary intake in American adults, NHANES 2005-2010. Int J Obes (Lond) 39, 820-827.
23. Bes-Rastrollo M, Basterra-Gortari FJ, Sanchez-Villegas A et al. (2010) A prospective study of eating away-from-home meals and weight gain in a Mediterranean population: the SUN (Seguimiento Universidad de Navarra) cohort. Public Health Nutr 13, 1356-1363.

24. Fulkerson JA, Farbakhsh K, Lytle L et al. (2011) Away-fromhome family dinner sources and associations with weight status, body composition, and related biomarkers of chronic disease among adolescents and their parents. J Am Diet Assoc 111, 1892-1897.

25. Bezerra IN, Souza Ade M, Pereira RA et al. (2013) Consumption of foods away from home in Brazil. Rev Saude Publica 47, Suppl. 1, 200S-211S.

26. Drewnowski A \& Rehm CD (2013) Energy intakes of US children and adults by food purchase location and by specific food source. Nutr J 12, 59.

27. Inagami S, Cohen DA, Brown AF et al. (2009) Body mass index, neighborhood fast food and restaurant concentration, and car ownership. J Urban Health 86, 683-695.

28. Mehta NK \& Chang VW (2008) Weight status and restaurant availability a multilevel analysis. Am J Prev Med 34, 127-133.

29. Plurphanswat N \& Rodu B (2014) The association of smoking and demographic characteristics on body mass index and obesity among adults in the US, 1999-2012. BMC Obes 1, 18.

30. Ouyang Y, Wang H, Su C et al. (2015) Use of quantile regression to investigate changes in the body mass index distribution of Chinese adults aged 18-60 years: a longitudinal study. BMC Public Health 15, 278.

31. Buttet S \& Dolar V (2014) Toward a quantitative theory of food consumption choices and body weight. Econ Hum Biol 17, 143-156.

32. Garawi F, Devries K, Thorogood N et al. (2014) Global differences between women and men in the prevalence of obesity: is there an association with gender inequality? Eur J Clin Nutr 68, 1101-1106.

33. Han E \& Powell LM (2011) Effect of food prices on the prevalence of obesity among young adults. Public Health 125, 129-135.

34. Kwon YS \& Ju SY (2014) Trends in nutrient intakes and consumption while eating-out among Korean adults based on Korea National Health and Nutrition Examination Survey (1998-2012) data. Nutr Res Pract 8, 670-678.

35. Razak F, Corsi DJ \& Subramanian SV (2013) Change in the body mass index distribution for women: analysis of surveys from 37 low- and middle-income countries. PLoS Med 10, e1001367. 